\title{
Scapula winging in a sports injury clinic
}

\author{
G. J. Packer FRCSEd, G. R. McLatchie FRCS* and W. Bowden FRCS
}

Departments of General and Orthopaedic Surgery, Hartlepool General Hospital, Hartlepool, Cleveland, UK, and ${ }^{*}$ National Sports Medicine Institute, London, UK

\begin{abstract}
Scapula winging is an uncommon condition but one which may be underdiagnosed. Four patients with scapula winging referred to a sports injury clinic are presented. None of the patients was aware of any trauma and a traction injury to the long thoracic nerve is proposed as the aetiology of this condition. These case reports emphasize the importance of excluding winging of the scapula in patients who present to sports injury clinics with shoulder pain.
\end{abstract}

Keywords: Scapula winging, sports injury clinic

Four patients from a variety of sports attended the clinic with scapula winging over an 18-month period and are presented below.

\section{Case reports}

\section{Case 1}

A 23-year-old right-handed boxer was referred with pain in the right shoulder of 8-10 weeks' duration. He had been training normally but there was no history of particular trauma. On examination he had winging of the right scapula. The winging recovered spontaneously over an 18-month period.

\section{Case 2}

A 50-year-old weightlifter presented with 1 year's history of an abnormal appearance of the right shoulder. There was no history of trauma. On examination he had winging of the right scapula. Electromyographic (EMG) studies demonstrated a neurogenic lesion of the serratus anterior in isolation. No recovery occurred over 1 year and he was discharged from follow-up.

\section{Case 3}

A 27-year-old right-handed squash player presented 6 months after a sudden onset of aching in the right shoulder and a loss of power in the right arm while

Address for correspondence: Mr G. J. Packer, Registrar in Orthopaedic Surgery, Royal Victoria Infirmary, Queen Victoria Road, Newcastle upon Tyne NE1 4LF, UK

(C) 1993 Butterworth-Heinemann Ltd 0306-3674/93/020090-02 playing squash. On examination (Figure 1) he had winging of the right scapula. This recovered spontaneously over a 6-month period.

\section{Case 4}

A 38-year-old right-handed rugby player experienced a sudden onset of pain in the right shoulder after a game of rugby, although he was unaware of injury during the game. Examination demonstrated scapula winging which recovered spontaneously over the next 6 months.

\section{Discussion}

Four cases of scapula winging were detected in a sports injury clinic over an 18-month period. Scapula winging is said to be a rare condition, the subject of some 250 case reports since first described in $1825^{1}$. The incidence among sportsmen may be higher, Gregg et al. ${ }^{1}$ detecting ten cases in a sports injury clinic over a 3-year period. Trauma has been the precipitating factor in many of the previous case reports ${ }^{2}$.

Scapula winging has been classified into static or dynamic types, and of bone, joint, muscle or nerve aetiology ${ }^{3}$. The cadaveric studies of Gregg et al. ${ }^{1}$ suggested that the most likely aetiology in the

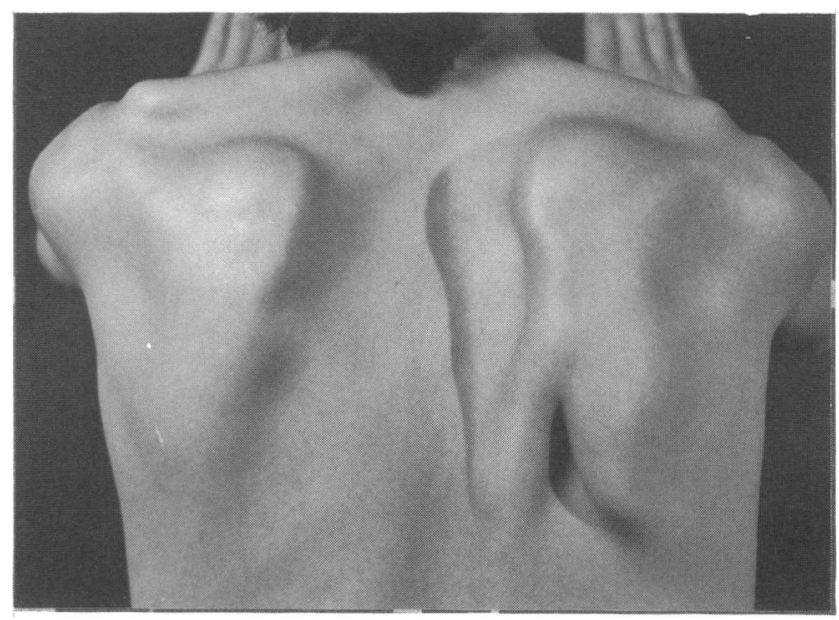

Figure 1. Scapula winging in Case 3 
absence of direct trauma was a traction injury to the long thoracic nerve which they were able to produce by rotation and flexion of the head plus a lateral tilt towards the contralateral shoulder while the ipsilateral shoulder was raised to an overhead position. That a traction injury resulting in a neurapraxia was the cause in the cases presented is supported by the spontaneous recovery of three of the four patients.

As the winging results from a neurapraxia, treatment consists of 'supervised neglect', shoulder movements being maintained under physiotherapy supervision. Surgery for this condition, e.g. pectoralis muscle transfer ${ }^{2}$, should be reserved for patients who show no signs of recovery clinically or on EMG studies after a suitable interval (18 months) for the neurapraxia to resolve.
The absence of precipitating trauma and the non-specific history may explain why scapula winging may be underdiagnosed. These case reports therefore emphasize the importance of considering scapula winging in patients presenting to sports injury clinics with shoulder pain or loss of function. Routine testing for scapula winging (by pushing against a wall with the shoulder flexed to $90^{\circ}$ ) should be performed in all patients with shoulder problems.

\section{References}

1 Gregg JR, Labosky D, Harty M et al. Serratus anterior paralvsis in the young athlete. J Bone Joint Surg $[\mathrm{Am}]$ 1979; 61-A: 825-32.

2 Gozna ER, Harris WR. Traumatic winging of the scapula. I Bone Joint Surg [Am] 1981; 61-A: 1230-33.

3 Fiddian NJ, King RJ. The winged scapula. Clin Orthop 1984; 185: $228-36$ 